\title{
Satisfacción de usuarias del control prenatal en Monteria
}

\section{Users' satisfaction with prenatal care in Monteria}

\author{
Luis-Enrique Mayorga-Mogollón'; Carlos-Enrique Sejín-Vásquez¹; Mileidys Pérez-Gómez' Jennifer Guzmán-Martínez
}

\begin{abstract}
Forma de citar: Mayorga-Mogollón LE, Sejín-Vásquez CE, Pérez-Gómez M, Guzmán-Martínez J. Satisfacción de usuarias del control prenatal en Montería. Rev Univ Ind Santander Salud. 2019; 51(3): $220-227$. doi: http://dx.doi.org/10.18273/revsal.v51n3-2019005 (c) (i)
\end{abstract}

\section{Resumen}

Introducción: En los procesos de atención que requieren seguimiento, como el control prenatal, la satisfacción de la usuaria puede contribuir a su resultado. La percepción de la calidad, estimada mediante la satisfacción de las usuarias, aproxima a la medición de la calidad de la atención prenatal. Objetivo: Establecer el nivel de satisfacción con el control prenatal en una institución prestadora de servicios de salud en Montería (Colombia). Metodología: Estudio transversal con componente analítico, utilizando el programa Epidat 3.1. Se estimaron promedios, medianas y proporciones según fuesen las variables cuantitativas o cualitativas. Se exploró la asociación estadística la prueba t de Student para las variables continuas y la prueba $\mathrm{x}^{2}$ para las variables categóricas, $\mathrm{y}$ se estimaron los OR (IC 95\%) asumiendo como caso la satisfacción con el control prenatal. Resultados: De las 178 mujeres entrevistadas 96\% manifestaron sentirse satisfechas con el control prenatal. Recibieron información sobre: la prueba del Virus de Inmunodeficiencia Humana 83,7\%, exámenes de laboratorio 85,4\%, signos de alarma 93,8\%, nutrición 86,5\%, lactancia $76,4 \%$, planificación $64 \%$, cuidados del recién nacido $71,9 \%$. Los promedios de edad (22,7 versus 25,1 años) y de semanas de gestación (11,1 versus 14,9 semanas) son menores en las mujeres satisfechas con el control prenatal (valor $\mathrm{p}<0,001$ ). Otras variables asociadas con la satisfacción en el control prenatal: entrega completa de medicamentos formulados (OR 8,6 IC 95\% 1,6-46,2), oportunidad en la entrega de resultados de los exámenes (OR 6 IC 95\% 1,2-28,9), e información suministrada sobre los signos de alarma (OR 20,3 IC 95\% 3,7-112) y nutrición (OR 5,3 IC 95\% 1,16-25,6). Conclusión: Además de la influencia de las variables demográficas y obstétricas y de los atributos de la calidad de la atención, es importante denotar cómo la información y la educación para la salud se asocian con la satisfacción con el control prenatal.

Palabras clave: Satisfacción del paciente; Atención prenatal; Mujeres embarazadas; Educación en salud; Calidad de la atención en salud; Educación prenatal; Servicios de salud materno-infantil; Encuesta de atención de la salud; Estudios transversales.

\begin{abstract}
Introduction: In the care health processes that require follow-up, such as prenatal care, users satisfaction can contribute to its outcome. The perceived quality, estimated through users satisfaction, approximates the measurement of prenatal care quality. Objective: To establish the level of satisfaction among pregnant women with prenatal care

1. Universidad del Sinú. Montería, Colombia.

Correspondencia: Luis Enrique Mayorga Mogollón. Universidad del Sinú, Carrera 1W 38-153 Barrio Juan XXIII Montería. Teléfono: +57 3205494460. Correo electrónico: mayorgaluise@gmail.com
\end{abstract}


in a health institution in Montería (Colombia). Methodology: Cross-sectional study with descriptive and analytical, using the Epidat 3.1 program. Averages, medians and proportions were estimated according to the quantitative or qualitative variables. The statistical association was explored using the Student's t test for the continuous variables and the $\mathrm{x}^{2}$ test for the categorical variables, and OR was estimated $(95 \% \mathrm{CI})$ assuming as a case the satisfaction with the prenatal control. Results: Of the 178 women interviewed, $96 \%$ reported feeling satisfied with antenatal consultation. They received information on: Human Immunodeficiency Virus test $83.7 \%$, laboratory tests $85.4 \%$, obstetric danger signs $93.8 \%$, maternal nutrition $86.5 \%$, breastfeeding $76.4 \%$, family planning $64 \%$, newborn care $71.9 \%$. The average of age (22.7 versus 25.1 years) and gestational age (11.1 versus 14.9 weeks) are lower in women satisfied with antenatal consultation $(\mathrm{p}<0.001)$. Other variables associated with satisfaction in antenatal care: complete medication delivery (OR 8.6 95\%CI 1.6-46.2), timely laboratory results (OR 6 95\% CI 1.2-28.9), and information provided on obstetric danger signs (OR 20.3 95\%CI 3.7-112) and maternal nutrition (OR 5.3 95\%CI 1.16-25.16). Conclusion: In addition to the influence of demographic and obstetric variables and attributes of quality care health, it is important to note how information and health education are also associated with women's satisfaction with antenatal care.

Keywords: Patient satisfaction; Prenatal care; Pregnant women; Health education; Quality of health care; Prenatal education; Maternal-child health services; Health care surveys; Cross-sectional studies.

\section{Introducción}

Montería, la capital del departamento de Córdoba, alberga al 25,8\% de la población cordobesa ${ }^{1}$. Algunos indicadores negativos de salud reproductiva de Córdoba en el año 2017 superaron los promedios nacionales, tales como la razón de mortalidad materna (69,4 versus 51,4 por 100.000 nacidos vivos en Colombia) ${ }^{2}$, la razón de morbilidad materna extrema (36,5 versus 35,8 por mil nacidos vivos en Colombia) ${ }^{3}$ y la tasa de mortalidad perinatal y neonatal tardía (21,1 versus 14,7 por 1.000 nacidos vivos en Colombia) ${ }^{4}$.

Además del reconocido impacto que el control prenatal tiene sobre los indicadores de mortalidad y de morbilidad maternas y perinatal, la información y la educación prenatales incrementan la calidad de vida de la madre durante el primer año después del parto ${ }^{5}$. El control prenatal incluye actividades orientadas a la prevención, detección y tratamiento oportunos de las condiciones que pudiesen ser peligrosas para la madre o para el feto ${ }^{6}$. Este conjunto de actividades también se propone educar a la gestante para optimizar su salud y para la maternidad y la crianza ${ }^{7}$.

Aunque la satisfacción de los usuarios reviste cierta abstracción conceptual de difícil medición ${ }^{8}$ y se le reconoce variabilidad histórica y cultural, si refleja dos elementos importantes en un servicio de salud: se relaciona con la calidad de la atención ${ }^{9}$ y con la adherencia a los planes de promoción de la salud, de prevención de la enfermedad y terapéuticos. En los procesos de atención que requieren seguimiento e intervenciones concomitantes, como en el control prenatal, la satisfacción de la usuaria reviste particular importancia por cuanto contribuye al resultado exitoso de su propósito ${ }^{10}$.

Algunos elementos del proceso de atención prenatal, como los largos tiempos de espera, se han relacionado con la no satisfacción de las usuarias ${ }^{11}$. No obstante, también se describe la ausencia de relación entre la calidad de la atención y la percepción y satisfacción de las usuarias ${ }^{12}$.

Un análisis de calidad de atención prenatal señala su asociación con infraestructura, equipo y talento humano $^{13}$. Sin embargo, se ha descrito que la medición de la calidad requiere evaluar procesos y resultados, puesto que la disponibilidad de insumos e infraestructura no se reflejan necesariamente en la calidad de la atención en salud ${ }^{14}$.

En consecuencia, la percepción de la calidad, estimada mediante la satisfacción de las usuarias de un servicio de control prenatal, es una arista de la aproximación a la medición de la calidad de la atención prenatal. Por tanto, este estudio se realizó con la finalidad de establecer el nivel de satisfacción de las gestantes con el control prenatal en una Institución Prestadora de Servicios de Salud (IPS) de la red pública de Montería.

\section{Metodología}

En septiembre, octubre y noviembre de 2017 se realizó, de manera anónima y mediante muestreo no probabilístico, por conveniencia, una encuesta de satisfacción a las mujeres gestantes usuarias del 
programa de control prenatal en una IPS del primer nivel de atención de la red pública de Montería (Colombia). La encuesta, estructurada, se aplicó en la sala de espera del servicio de consulta externa. La encuesta fue diligenciada por estudiantes de octavo semestre de medicina. Se excluyeron las mujeres gestantes que realizaban su control prenatal en otra IPS.

La encuesta se dividió en cuatro dimensiones: a) información demográfica y obstétrica, constituida por las variables edad, régimen de afiliación al sistema de salud, tipo de consulta (primera vez o control), semanas de gestación al iniciar el control prenatal, b) aspectos de oportunidad en el proceso de atención, conformada por las variables entrega completa de medicamentos formulados en la consulta de control prenatal, tiempo para la autorización de exámenes paraclínicos del segundo nivel, resultados de los exámenes disponibles para la siguiente cita, c) características de la información suministrada a la usuaria, integrada por la variables de información sobre exámenes de laboratorio, la prueba VIH, signos de alarma, lactancia, anticoncepción, nutrición, cuidados del recién nacido, cáncer de cuello uterino, autoexamen de seno e importancia que la pareja le acompañase a la consulta de control prenatal y d) satisfacción de la gestante con el control prenatal, mediante una pregunta sobre si estaba o no satisfecha. Se les indagó sobre las variables en relación con su última consulta de control prenatal.

Con base en una proporción esperada de satisfacción con el control prenatal de $80 \%$, análoga a la encontrada en otra ciudad de la región caribe ${ }^{10}$, y a partir de la expresión $n=\left(Z^{2} p q / e^{2}\right)$, con $Z 1,96, p$, 0,8 , q 0,2 , error 0,06 , se estimó en 170 participantes el tamaño de la muestra.

Análisis estadístico: Para realizar este estudio transversal con componente analítico se elaboró la base de datos en la hoja de cálculo Excel y se analizó el comportamiento de las variables en distintos estratos utilizando el programa Epidat 3.1. Se estimaron promedios, medianas y proporciones según fuesen las variables cuantitativas o cualitativas. Se exploró la asociación estadística mediante la prueba $\mathrm{t}$ de Student para las variables continuas y la prueba de $\mathrm{x}^{2}$ para las variables categóricas, y se estimaron los OR (IC 95\%) asumiendo como caso la satisfacción con el control prenatal y como exposición las siguientes circunstancias o intervenciones: consulta de control, entrega completa de los medicamentos, oportunidad en los resultados de los exámenes de laboratorio, tiempo para la autorización (en la Entidades
Promotoras de Salud - EPS) de los exámenes de segundo nivel, y el suministro de información sobre la importancia que la pareja la acompañase en las consultas de control prenatal, sobre la prueba VIH (Virus de Inmunodeficiencia Humana), los exámenes de laboratorio, los signos de alarma, la nutrición de la gestante, lactancia, planificación, cuidados del recién nacido, cáncer de cérvix (citología cervicouterina) y cáncer de mama (autoexamen de seno).

\section{Resultados}

Se entrevistaron 178 mujeres gestantes. De ellas, 171 (96\%) manifestaron sentirse satisfechas con la atención recibida en el control prenatal. La edad promedio fue 22,8 años (rango 14 a 36 años, desviación estándar 4,4), mediana 23 años (Tabla 1). El promedio de semanas de gestación al iniciar el control prenatal fue 11,4 semanas (rango 4-31; desviación estándar 6,2; mediana 9 semanas).

El promedio de edad de las mujeres satisfechas con el control prenatal es menor (22,7 versus 25,1 años) que en las gestantes no satisfechas $(\mathrm{p}<0,001)$. Menos de la quinta parte de las usuarias estaba iniciando su control prenatal (Tabla 1). No se halló diferencia en el nivel de satisfacción entre quienes consultaban por primera vez o estaban en una consulta de control (OR 0,74 IC95\% 0,08$6,41)$. El promedio de semanas de gestación al iniciar el control prenatal de quienes se manifestaron satisfechas es menor (11,1 versus 14,9 semanas de gestación) que el de los siete casos de no satisfacción $(\mathrm{p}<0,001)$.

En más de la mitad de los casos (54\%) la autorización de la EPS para exámenes de segundo nivel se efectuó en tres días o menos (Tabla 2), pero el nivel de satisfacción es independiente del tiempo de tres o menos días para autorización de los exámenes de segundo nivel. Sin embargo, la entrega oportuna de los resultados de los exámenes paraclínicos para que estuviesen disponibles la siguiente cita y la completitud de la entrega de los medicamentos formulados durante la consulta de control prenatal sí se asocian con el nivel de satisfacción.

En la Tabla 3 se observa la distribución porcentual de la información suministrada sobre la prueba VIH, exámenes de laboratorio, signos de alarma en la gestante, nutrición de la gestante, importancia del autoexamen de seno, cómo prepararse para la lactancia, métodos para planificar y cuándo comenzar, cuidados del recién nacido o cómo prepararse para la crianza, importancia de la citología de cérvix, e importancia que la pareja le acompañase a las consultas del control prenatal. 
Tabla 1. Características demográficas y obstétricas de las mujeres participantes en el estudio.

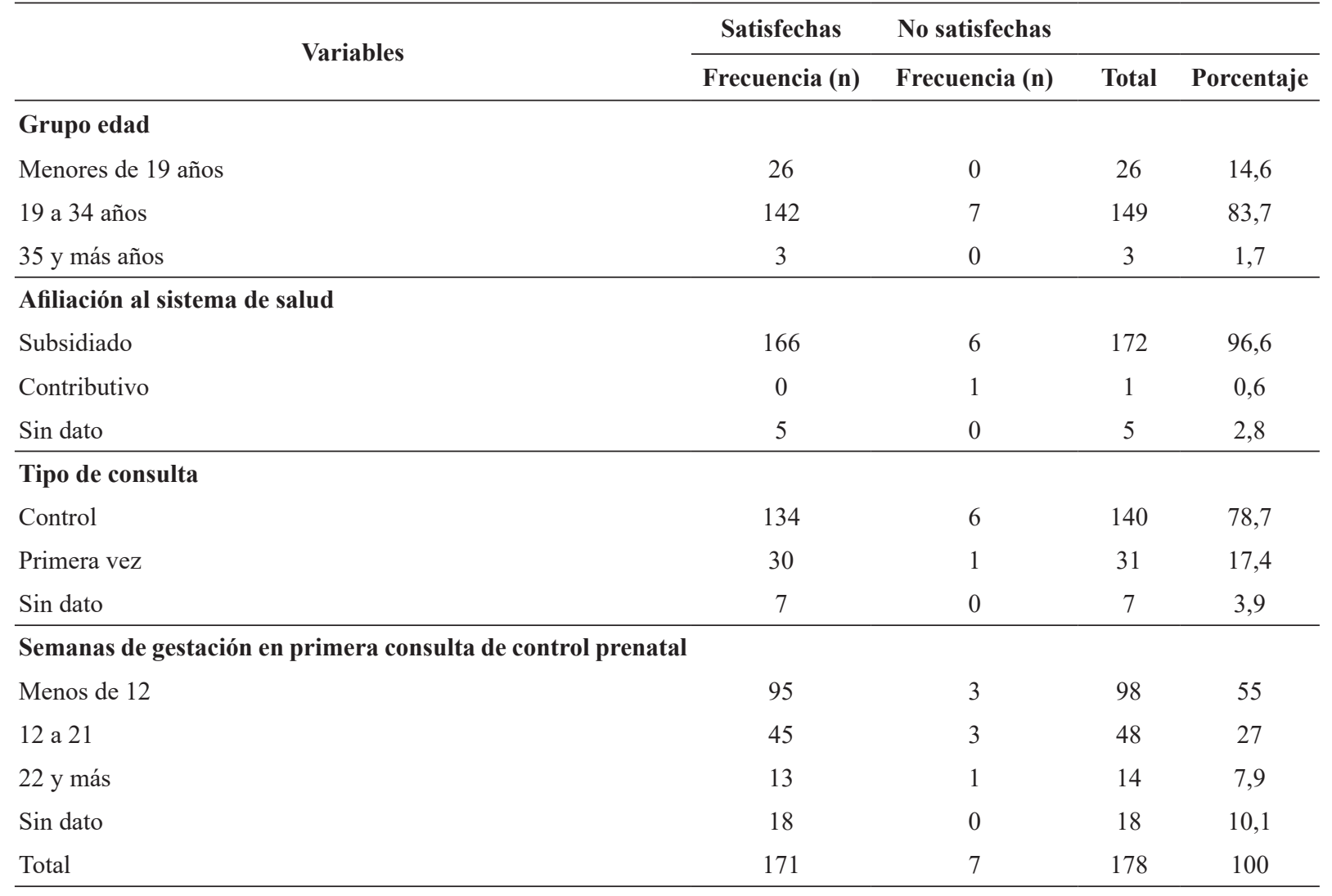

Tabla 2. Indicadores de oportunidad reportados por las participantes en el estudio.

\begin{tabular}{|c|c|c|c|c|c|}
\hline \multirow{2}{*}{ Variables } & Satisfechas & No satisfechas & & & \\
\hline & Frecuencia (n) & Frecuencia (n) & Total & Porcentaje (\%) & OR (IC 95\%) \\
\hline
\end{tabular}

Entrega completa de medicamentos

\begin{tabular}{lccccc} 
Sí & 147 & 3 & 150 & 84,3 & $8,6(1,6-46,2)$ \\
No & 17 & 3 & 20 & 11,2 & \\
No aplica & 5 & 1 & 6 & 3,4 & \\
Sin dato & 2 & 0 & 2 & 1,1 & \\
\hline Tiempo de autorización (exámenes segundo nivel) & & & & & \\
Mismo día & 44 & 1 & 45 & 25,3 & \\
1 a 3 días & 49 & 2 & 51 & 28,7 & $1,6,3-7,8)$ \\
1 a 3 semanas & 54 & 2 & 56 & 31,5 & \\
4 semanas o más & 19 & 2 & 21 & 11,8 & \\
Sin dato & 5 & 0 & 5 & 2,8 & \\
\hline Resultados de exámenes para la siguiente cita & & & & & \\
Sí & 144 & 4 & 148 & 83,1 & $6(1,2-28,9)$ \\
No & 18 & 3 & 21 & 11,8 & \\
No aplica & 4 & 0 & 4 & 2,3 & \\
Sin dato & 5 & 0 & 5 & 2,8 & \\
Total & 171 & 7 & 178 & 100 & \\
\hline
\end{tabular}


Tabla 3. Información suministrada.

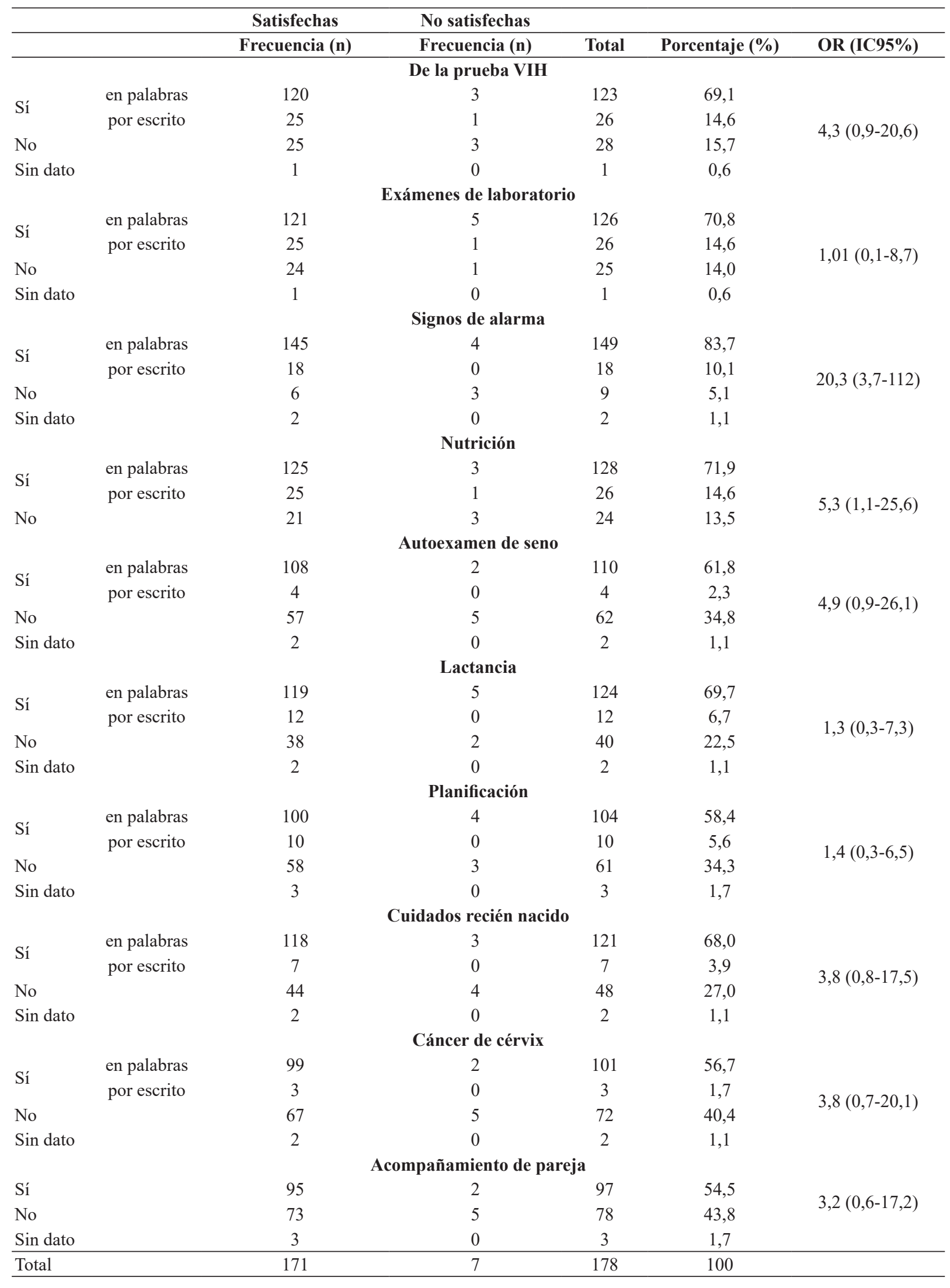


De las 123 mujeres que recibieron asesoría pre test VIH de manera verbal, 95,1\% refirieron que sí entendieron la información suministrada; en seis casos no se registró la respuesta. Asimismo, de las 26 mujeres que recibieron la información por escrito, 76,9\% manifestaron que sí entendieron la información, 11,5\% respondieron que no entendieron la información y en tres casos no se registró la respuesta.

De las 126 gestantes que recibieron información verbal sobre exámenes de laboratorio, $92 \%$ respondieron que sí entendieron la información suministrada, solo un caso expresó que no entendió la información, y en 11 casos no se registró la respuesta. De las 26 mujeres que recibieron información escrita sobre los exámenes de laboratorio, 96,2\% sí entendieron la información, en un caso no se registró la respuesta, ninguna mujer relató no haber entendido la información escrita.

De las 149 gestantes que recibieron información verbal sobre signos de alarma, 91,3\% relataron que sí comprendieron la información suministrada, en 12 casos no se registró la respuesta. De las 18 mujeres que recibieron la información escrita sobre signos de alarma $94,4 \%$ dijeron que sí la comprendieron, en un caso no se registró la respuesta.

De las 128 mujeres que recibieron información verbal sobre nutrición de la gestante $94,5 \%$ respondieron que sí entendieron la información dada, en siete casos no se registraron las respuestas. De las 26 gestantes que recibieron información por escrito $80,8 \%$ respondieron que sí entendieron la información sobre lo que es recomendable comer, una gestante manifestó que no entendió la información dada, y en cuatro casos no se registró la respuesta.

De las 124 gestantes que recibieron información verbal acerca de cómo prepararse para la lactancia $88,7 \%$ dijeron que sí entendieron la información suministrada. De las 12 mujeres que recibieron información escrita sobre la lactancia $75 \%$ expresaron que sí entendieron la información.

De las 104 mujeres que recibieron información verbal sobre métodos para planificar y cuándo comenzar $85,6 \%$ relataron que sí entendieron la información suministrada. De las diez mujeres que recibieron información escrita nueve dijeron que sí entendieron la información.

De las 121 mujeres que recibieron información verbal sobre cómo prepararse para la crianza $84,3 \%$ expresaron que sí entendieron la información suministrada, tres manifestaron no haber entendido la información, y en $13,9 \%$ de los casos no se registró la respuesta. De las siete mujeres que recibieron información escrita sobre cuidados del recién nacido, cinco comprendieron la información, una manifestó que no entendió y en un caso no se registró la respuesta.

De las 101 gestantes que recibieron información sobre la importancia de la citología de cérvix, $74,6 \%$ sí comprendieron la información suministrada, 9,9\% no entendieron la información recibida y en $15,8 \%$ de los casos no se registró la respuesta. De las tres mujeres que recibieron información por escrito sobre la importancia de la citología cervicouterina, dos manifestaron que sí la comprendieron y una dijo que no la entendió.

De las 110 mujeres que recibieron información sobre la importancia del autoexamen del seno $89,1 \%$ sí entendieron la información, en $9,1 \%$ de casos no se registró la respuesta. De las cuatro mujeres que recibieron información escrita sobre la importancia del autoexamen de seno, dos manifestaron que sí la entendieron, una respondió que no la entendió y en un caso no se registró la respuesta.

En síntesis, la satisfacción en el control prenatal se asocia con: edad, inicio temprano del control prenatal, la entrega completa de los medicamentos formulados, la oportunidad en la entrega de los resultados de los exámenes, y la información suministrada sobre los signos de alarma, y nutrición de la gestante.

\section{Discusión}

Si bien la valoración de la satisfacción de usuarios de servicios de salud contiene dificultades de comparabilidad $^{15}$, debido a la dependencia de las expectativas de los usuarios, que si fuesen bajas pueden resultar satisfechos al recibir servicios relativamente deficientes $^{16}$, a la variabilidad en los instrumentos y en los indicadores, y a que es un concepto que se enmarca en una noción multidimensional, el alto nivel de satisfacción con el control prenatal (96\%) en la IPS de primer nivel de la red pública de Montería es superior al hallado en estudios de otras ciudades del caribe colombiano, como el realizado en instituciones de salud de primer nivel en Cartagena $(84 \%)^{10}$ o el de Sincelejo en el que los satisfactores de los prestadores del servicio de control prenatal, identificados por las gestantes, oscilan alrededor de $90 \%{ }^{17}$.

El promedio de edad es similar al estimado en los mencionados estudios de Cartagena ${ }^{10}$ y Sincelejo ${ }^{17}$ $y$ al realizado en Cali y Manizales ${ }^{18}$. No obstante, en 
este estudio sí es más alta la proporción de afiliación al régimen subsidiado $(96,6 \%)$ que las de los citados: alrededor de $80 \%$ en el de Sincelejo ${ }^{17}$ y de $70 \%$ en el de Cali y Manizales ${ }^{18}$.

La asociación con la menor edad se contrapone con los hallazgos de estudios de Italia ${ }^{19}$, que refiere mayor nivel de satisfacción con la atención prenatal a mayor edad de la gestante, y de Nigeria $^{20}$ que describe la ausencia de asociación de la edad materna con la satisfacción en el control prenatal.

De otra parte, la relación establecida con el inicio temprano del control prenatal podría ir en el mismo sentido de lo hallado en países africanos, donde la atención prenatal de menor calidad se encontró en las mujeres que ingresan con más de 24 semanas de gestación ${ }^{13}$.

De manera similar a lo descrito en Costa Rica ${ }^{21}$ y en México $^{22}$, las variables oportunidad en resultados de exámenes y entrega completa de los medicamentos formulados se asocian con la satisfacción. También un estudio transversal en Kenia relaciona la mayor satisfacción de los usuarios de los servicios de salud con el trato personal de salud-usuario y menores tiempos de espera ${ }^{23}$.

La asociación establecida entre la satisfacción con la atención prenatal y la información suministrada sobre signos de alarma y sobre nutrición de la gestante se constata con lo hallado en un ensayo clínico ${ }^{5}$ en el cual se muestra que se obtiene mayor nivel de felicidad y calidad de vida en las mujeres que reciben educación prenatal, la cual incluyó información sobre cambios anatómicos, fisiológicos y psicológicos durante el embarazo, nutrición durante el embarazo, cuidados del recién nacido y de la madre en el postparto, lactancia y planificación.

Aunque la satisfacción de las mujeres con la atención prenatal resulte del equilibrio entre sus expectativas y la atención que reciben en los servicios de salud, se corrobora que además de la influencia de las variables demográficas y obstétricas y de los atributos de la calidad de la atención como la oportunidad, también el suministro de información y la educación para la salud se asocian con la satisfacción de las gestantes con el control prenatal.

\section{Consideraciones éticas}

De acuerdo con el artículo 11 de la resolución 8430 de 1993, es una investigación sin riesgo puesto que es un estudio en el que no se realizó ninguna intervención o modificación intencionada de las variables biológicas, fisiológicas, sicológicas o sociales de los individuos que participaron en el estudio, entre los que se consideran: entrevistas, cuestionarios y otros en los que no se les identifique ni se traten aspectos sensitivos de su conducta $^{24}$.

\section{Conflicto de interés}

Los autores declaran que no hay conflicto de intereses en el presente manuscrito.

\section{Referencias}

1. Departamento Administrativo Nacional de Estadística. Estimación y proyección de población nacional, departamental y municipal total por área 1985-2020.

2. Instituto Nacional de Salud. Mortalidad materna Colombia; 2017.

3. Instituto Nacional de Salud. Morbilidad materna extrema Colombia; 2017.

4. Instituto Nacional de Salud. Mortalidad perinatal y neonatal tardía; 2017.

5. Bahrami N, Simbar M, Bahrami S. The effect of prenatal education on mother's quality of life during first year postpartum among Iranian women: a randomized controlled trial. Int J Fertil Steril. 2013; 7(3): 169-174.

6. Ministerio de salud y protección social. Colciencias. Guías de práctica clínica para la prevención, detección temprana y tratamiento del embarazo, parto o puerperio. Bogotá: Ministerio de salud y protección social-Colciencias, 2013.

7. Cáceres-Manrique FM. El control prenatal: una reflexión urgente. Rev Colomb Obstet Ginecol. 2009; 60(2): 165-170.

8. Jiménez Pérez JM, García Villanueva S. Evaluación de la satisfacción del usuario del sistema nacional de salud en el último quinquenio. Rev Enferm. CyL 2015; 7(2): 21-34.

9. Reynaldos-Grandón K, González-Pinilla G, Guerra-Díaz X, Gutiérrez-Fuenzalida F, Santibáñez-Muñoz L. Factores mal evaluados en encuestas de satisfacción aplicadas a usuarios de atención primaria en Latinoamérica. Rev Enferm Act Costa Rica. 2017; 33. doi: 10.15517/REVENF.V0I33.29156.

10. Castillo I, Villarreal M, Olivera E, Pinzón A, Carrascal H. Satisfacción de usuarias del control prenatal en instituciones de salud públicas y factores asociados. Cartagena. Hacia Promoc Salud. 2014; 19(1): 128-140. 
11. Do M, Wang W, Hembling J, Ametepi P. Quality of antenatal care and client satisfaction in Kenya and Namibia. Int J Qual Health Care. 2017; 29(2): 183193. doi: 10.1093/intqhe/mzx001.

12. Diamond-Smith N, Sudhinaraset M, Montagu D. Clinical and perceived quality of care for maternal, neonatal and antenatal care in Kenya and Namibia: the service provision assessment. Reprod Health. 2016; 13(1): 92. doi: 10.1186/s12978-016-0208-y.

13. Kruk ME, Chukwuma A, Mbaruku G, Leslie $\mathrm{HH}$. Variation in quality of primary-care services in Kenya, Malawi, Namibia, Rwanda, Senegal, Uganda and the United Republic of Tanzania. Bull World Health Organ. 2017; 95(6): 408-418.

14. Leslie HH, Sun Z, Kruk ME. Association between infrastructure and observed quality of care in 4 healthcare services: A cross-sectional study of 4,300 facilities in 8 countries. PLoS Med. 2017; 14(12). doi: 10.1371/journal.pmed.1002464.

15. Tiga-Loza D, Villar-Centeno L, Güiza-Sanabria D, Martínez-Vega R. Validez y confiabilidad de un instrumento de satisfacción del usuario con síndrome febril agudo. Rev Salud Pública. 2010; 12(5): 820-832.

16. Zamora S. Satisfacción sobre la infraestructura y la calidad de atención en la consulta de GinecoObstetricia de un establecimiento de atención primaria. Horiz. Med. 2016; 16 (1): 38-47.

17. Miranda-Mellado C. Factores de capacidad en el uso de control prenatal adecuado en gestantes de Sincelejo (Colombia). Salud Uninorte. 2016; 32 (3): 436-451.

18. Rivera P, Carvajal R, Mateus J, Arango F, Valencia J. Factores de servicios de salud y satisfacción de usuarias asociados al acceso al control prenatal. Hacia Promoc Salud. 2014; 19(1): 84-98.

19. Tocchioni V, Seghieri C, De Santis G, Nuti S. Sociodemographic determinants of women's satisfaction with prenatal and delivery care services in Italy. Int J Qual Health Care. 2018; 30(8): 594-601. doi: 10.1093/intqhe/mzy078.

20. Oladapo OT, Osiberu MO. Do sociodemographic characteristics of pregnant women determine their perception of antenatal care quality? Matern Child Health J. 2009; 13: 505-511. doi: 10.1007/s10995-008-0389-2.

21. Moraga H, González R. Satisfacción del usuario que requiere exámenes de laboratorio urgentes, Costa Rica. Rev Costarric Salud Pública. 2011; 20(1): 44-48.

22. Vera J, Trujillo A. El efecto de la calidad del servicio en la satisfacción del derechohabiente en instituciones públicas de salud en México.
Contad Admin. 2018; 63(2): 1-22. doi: http://dx.doi.org/10.1016/j.cya.2016.07.003.

23. Wambua JM, Mbayaki R, Munyao PM, Kabue MM, Mulindi R, Change PM, et al. Client satisfaction determinants in four Kenyan slums. Int $\mathrm{J}$ Health Care Qual Assur. 2015; 28(7): 667-677. doi: 10.1108/IJHCQA-12-2014-0110.

24. Ministerio de Salud de Colombia. Resolución 8430 de 1993. Por la cual se establecen las normas científicas, técnicas y administrativas para la investigación en salud. 